\title{
Mir-129-2-3p Has Tumor Suppressor Role in Ewing Sarcoma Cell Lines and Cancer Tissue Samples
}

Esra Guzel Tanoglu1,2*

https://orcid.org/0000-0002-0909-8935

Yavuz Arıkan ${ }^{3}$

https://orcid.org/0000-0001-7108-9864

Yavuz Selim Kabukcuoglu ${ }^{3}$

https://orcid.org/0000-0002-5976-1977

\author{
Fevziye Kabukcuoglu 4 \\ https://orcid.org/0000-0002-7705-7510
}

Alpaslan Tanoglu $\mathbf{u}^{5,6}$

https://orcid.org/0000-0002-7477-6640

Sukru Ozturk ${ }^{7}$

https://orcid.org/0000-0002-8809-7462

\begin{abstract}
${ }^{1}$ University of Health Sciences, Institute of Health Sciences, Department of Molecular Biology and Genetics, Istanbul, Turkey; ${ }^{2}$ University of Health Sciences, Experimental Medicine Research and Application Center, Istanbul, Turkey; ${ }^{3} \mathrm{MS}$ Baltalimani Bone Diseases Training and Research Hospital, Department of Orthopedics and Traumatology, Istanbul, Turkey; ${ }^{4}$ Sisli Etfal Training and Research Hospital, Department of Pathology, Istanbul, Turkey; ${ }^{5}$ University of Health Sciences, Sancaktepe Şehit Prof. Dr. Illhan Varank Training and Research Hospital, Department of Internal Medicine, Istanbul, Turkey; ${ }^{6}$ University of Health Sciences, Institute of Health Sciences, Department of Molecular Oncology, Istanbul, Turkey; ${ }^{7}$ Istanbul University, Istanbul Medical Faculty, Department of Internal Medicine, Division of Medical Genetics, Istanbul, Turkey.
\end{abstract}

Editor-in-Chief: Alexandre Rasi Aoki

Associate Editor: Daniel Fernandes

Received: 2021.05.11; Accepted: 2021.07.21.

*Correspondence: esra.guzel@sbu.edu.tr; Tel.: +90-216-4189616 (E.G.T.).

\section{HIGHLIGHTS}

- Ewing sarcoma (EWS) is the second most seen type of bone tumor in childhood.

- microRNAs may have regulatory effects in regulating tumor process of EWS.

- We have shown that regulatory role of miR-129-2-3p in EWS cell lines and human tissue samples.

- $\quad$ miR-129-2-3p acts as a tumor suppressor and reduced proliferation, migration, invasion in EWS.

Abstract: Deregulation of miRNA expressions was identified as a novel feature of tumor biology in Ewing sarcoma (EWS). The aim was to evaluate the regulatory role of miR-129-2-3p in EWS cell lines and human EWS tissue samples. EWS cell lines TC-71, TC-106, and CHLA-99 were used in the study and real-time PCR was utilized to investigate the functional role of tumor suppressor mir-129-2-3p and miR-129-2-3p levels in the cells. Proliferation, migration, invasion and apoptosis assays were carried out within the scope of functional in vitro studies. Expression levels of CDK6 and SOX4, which are miR-129-2-3p target genes, were examined. Moreover, the change in expression levels of miR-129-2-3p in EWS tumor tissues was also examined. It was determined that miR-129-2-3p expression markedly diminished in all the studied cell lines. In addition, miR-129-3p was found to decrease in proliferation, migration, invasion and apoptosis assays in all EWS cell lines. CDK6 and SOX4 levels were also decreased in miR-129-2-3p transfected cell lines. It was found that miR-129-2-3p levels were significantly decreased in EWS tumor tissue samples compared to the corresponding adjacent normal tissue samples. In line with the results of our current study, where the possible 
function of miR-129-2-3p in EWS cell lines was examined, for the first time in the literature miR-129-2-3p was shown to have low expression level in EWS lines and EWS tumor tissue samples, and to provide a tumor suppressor effect.

Keywords: Ewing Sarcoma; microRNA; biomarker; miR-129-2-3p; cell line.

\section{INTRODUCTION}

Ewing sarcoma (EWS) is the second most commonly seen type of bone tumor in childhood and the third most common type of osteosarcoma and chondrosarcoma at all ages. EWS consists of small round sarcoma cells and the tumor grows rapidly. Tumors in bone and soft tissues usually involve long bones, such as arms and legs [1].

Identifying EWS patients in the early period is important for developing better treatment and surveillance strategies to increase the late survival rate. It was suggested that explaining EWS tumor biology in a detailed manner may secure favorable survival rates in these patients [2]. In the literature, recent comprehensive studies showed the predictive values of many kind of biomarkers, especially for tumor diagnosis and prognosis. Nevertheless, there are still many possible diagnosis- and prognosis-associated candidate biomarkers that require further studies in EWS tumor biology [3].

miRNAs are 18-24 nucleotides in length, single-stranded, non-protein-encoding small RNA molecules. MiRNAs, which act as gene regulators, are located in the intergenic regions and in the exonic and intronic regions of protein-coding genes [4]. Currently, miRNAs were suggested to be pivotal regulators of numerous normal cellular events such as proliferation, growth, differentiation, and apoptosis. Despite the fact that miRNAs are indispensable for normal cellular homeostasis, a huge amount of miRNA studies suggest that miRNA expressions are deregulated in many kind of neoplasms and can influence aggressive, metastatic disease $[5,6]$.

The miR-129 family is one of the well-known cancer-related miRNAs. miR-129-2 belongs to this miRNA family and changes in expression levels of miR-129-2 was investigated in many kinds of solid tumors such as glioblastoma, lung and esophageal carcinoma. In these solid tumors it was mentioned as tumor suppressor miRNA [7-10]. Anyway, the role of miR-129-2-3p in EWS remains dark. In other words, its regulatory effects were not examined in EWS patients.

In this research, we aimed to investigate the regulatory role of miR-129-3p in EWS cell lines (TC-71, TC106 and CHLA-99 cells) and human EWS tissue samples. In other words, we aimed to evaluate the oncogenic or tumor suppressor and regulatory role of miR-129-2-3p. In this current study, we found that miR-129-2-3p acts as a tumor suppressor miRNA and reduced proliferation, migration, invasion, and colony capacity of EWS cells. Moreover, it induced tumor cell apoptosis and expression levels were also changed in human EWS tumor tissue samples.

\section{MATERIAL AND METHODS}

\section{EWS Samples}

A total of 31 Ewing's sarcoma/PNET and 12 corresponding adjacent normal tissues were obtained from Baltalimani Bone and Joint Diseases Education and Research Hospital. Patients were enrolled in the research after signing written informed consent. This study was approved by an institutional review board (Approval number: 2016/1293).

\section{RNA Isolation}

TC-71, TC-106 and CHLA-99 cell lines (from Children's Oncology Group) were seeded in complete medium in separate plates until $80 \%$ confluence was reached, followed by incubation in medium with $10 \%$ fetal bovine serum for 3 days. Total RNA was extracted from cell lines with TRIzoL and FFPE tissues using "mirVana PARIS kit" (Ambion, Darmstadt, Germany) according to the manufacturer's instructions. RNA concentration and purity were tested by Thermo Scientific ${ }^{\mathrm{TM}}$ NanoDrop ${ }^{\mathrm{TM}}$ 2000/2000c Spectrophotometers.

\section{cDNA synthesis and quantitative real-time PCR (qRT-PCR) procedure}

Total RNA samples of $30 \mathrm{ng} / \mathrm{ul}$ were used for miRNA expression analysis. The hsa-mir-214-3p and RNU43 specific primers were obtained from Applied Biosystems and TaqMan MicroRNA reverse transcription kit (Applied Biosystems). In order to determine miRNA expression levels, TaqMan Universal 
Master Mix (Applied Biosystems) was used according to the protocol. MiRNA expression data were normalized to RNU43.

During gene expression analysis, $500 \mathrm{ng}$ total RNA was used for cDNA reverse transcription using 'Transcriptor First-Strand cDNA Synthesis Kit' (Roche). cDNA of 1-5 uL was used for qRT-PCR using SYBR Green PCR Master Mix (Applied Biosystems) for CDK6 and SOX4. The CDK6 primers were sense: 5'GGATAAAGTTCCAGAGCCTGGAG-3' and antisense: 5'-GCGATGCACTACTCGGTGTGAA-3', SOX4 primers were sense: 5'-ACACTGGTGGCAGGTTAAGG-3' and antisense: 5'CGCTGTTTGGATTTCCTGAT-3' GAPDH primers were sense: 5'-GCCTCGCCTTTGCCGATC-'3 and antisense: 5'-CCCACGATGGAGGGGAAG-'3 with an annealing temperature of $60^{\circ} \mathrm{C}$. Each experiment was performed in duplicate using the LightCycler ${ }^{\circledR} 480$ Instrument. The variances in expression levels were tested using delta delta CT method. $\beta$-actin was used as an internal control.

\section{Cell culture and miRNA transfection}

EWS cell lines TC-71, TC-106, and CHLA-99, 10\% fetal bovine serum (FBS), 1\% penicillin, streptomycin, ampicillin (PSA) and L-glutamine on IMDM medium at $37^{\circ} \mathrm{C}$ were cultured in a humidified $5 \% \mathrm{CO} 2$ incubator. Mature miR-214-2-3p mimic and non-targeting mimic control were obtained from Invitrogen. A total of 300,000 cells were seeded into 6-well plates and cultured for $24 \mathrm{~h}$ before transfection with siRNA using Lipofectamine $^{\mathrm{TM}} 2000$ Transfection Reagent (Invitrogen) following the manufacturer's order.

\section{Cell proliferation assay}

A total of $5 \times 10^{3}$ cells were plated in each well of 96-well plates and attached cells were counted using the Cell Counting Kit-8 Cell Proliferation Assay Kit (Dojindo Molecular Technologies) with Beckman Cell counter. The absorbance was read at $450 \mathrm{~nm}$ and the experiment was repeated three times.

\section{Migration and invasion assay}

Boyden chamber (Corning) inserts were used for the invasion assay. Cell invasion assay was accomplished between the lower and upper chambers using BD BioCoat Matrigel invasion chambers (Becton Dickinson). After transfection with miR-214-2-3p or non-targeting miRNA, cells were seeded into invasion chambers at a density of $3 \times 10^{5}$ cells per chamber in triplicate. Medium containing FBS was placed on the bottom surface of the inserts and the cells were kept in the incubator for 24 hours to examine the migration and invasion potential of the EWS cell lines. The next day, the cells transferred to the lower surface of the inserts were fixed with $100 \%$ methanol, and stained with $0.3 \%$ crystal violet in $2 \%$ ethanol. After washing with phosphate buffered saline, the analysis process was carried out by counting the visible cells in the inserts.

\section{Caspase assay}

TC-71, TC-106 and CHLA-99 cells were seeded at 3000 cells per well into 96-well plates in duplicate separately. Cells transfected with mir-129-2-3p were examined 24 hours later for caspase-3 activity using the "CaspACETM Assay System, Colorimetric (Promega)" kit, according to the manufacturer's protocol. Determination of the apoptotic levels of the cells was carried out with absorbance measurements at $405 \mathrm{~nm}$ with the Multiskan FC micro plate reader (Thermo, Rockford USA).

\section{Soft-agar growth assay}

Six-well plates with $1.2 \%$ low melting agar mixed with $2 X$ culture media plus $20 \%$ FBS and $2 \%$ PSA were prepared before the seeding of EWS cell lines. EWS cells $\left(3 \times 10^{3}\right)$ transfected with mir-124-2-3p or nontargeting controls were plated in $0.35 \%$ agar layer in each agar dish. Cell line colonies were counted after incubation at $37^{\circ} \mathrm{C}$ in an incubator for 3 weeks and staining with crystal violet. This experiment was repeated in triplicate.

\section{In silico MicroRNA target prediction}

To pick out the putative targets of mir-129-2-3p, target prediction bioinformatics algorithms were used. miRanda http://www.microrna.org, mirBase http://www.mirbase.org and TargetScan $\mathrm{http}: / /$ www.targetscan.org algorithms were used to determine the putative targets of mir-129-2-3p and SOX4 and CDK6 were chosen. 


\section{Statistical Analysis}

All data were tested by using IBM SPSS, version 20. A "p" value of 0.05 or below was accepted as statistically significant. Data were plotted as mean \pm standard deviation and $p$ value was calculated from Student's t-test. Receiver operating characteristic (ROC) curves were plotted by using SPSS 20 to see the power of mir-129-2-3p to differentiate the EWS tumor samples from corresponding adjacent normal tissue samples.

\section{RESULTS}

\section{Proliferation, Migration-Invasion, Colony and Caspase Assay Results}

We showed downregulation of mir-129-2-3p expression level with proliferation, invasion, migration and soft agar colony assay in EWS cell lines. EWS cell lines (TC71, TC106 and CHLA99) had mir-129-2-3p analysis performed using qRT-PCR in cells. The qRT-PCR analysis showed that the mir-129-2-3p expression level is downregulated in EWS cells with a p value less than 0.01 (data not shown).

As a result of the proliferation assay experiments, a significant decrease in the proliferation rate of the cells transfected with mir-129-2-3p was found on the 3rd day compared to the control group have been shown in Figure 1a, 1b, 1c. (TC71 p<0.01, TC106 p<0.01, CHLA99 $p<0.001)$. It was found that mir-129-2-3p was effective in reducing migration and invasion capacities in TC-71, TC106, and CHLA99 cells (migration assay: TC71 $\mathrm{p}<0.001$, TC106 $\mathrm{p}<0.01$, CHLA99 $\mathrm{p}<0.001$ ) (Figure 1d, 1e, 1f) (invasion assay: TC71 $\mathrm{p}<0.01$, TC106 $p<0.05$, CHLA99 $p<0.01$ ). (Figure 1g, 1h, 1i). When the soft agar colony assay, which was done to understand the effect of mir-129-2-3p on the colony-forming capacity of EWS cells, was examined, the colony capacity of the 3 cell lines decreased compared to the control group (TC71, TC106, CHLA99 p<0.001), (Figure 2a). The caspase-3 activity assay showed that the increased expression of miR-129-2-3p in EWS cells caused increased in the caspase- 3 activity compared with non-targeting miRNA cells, respectively (TC71 $p<0.05$, TC106 $p<0.05$, CHLA99 $p<0.01$ ), (Figure 2b)
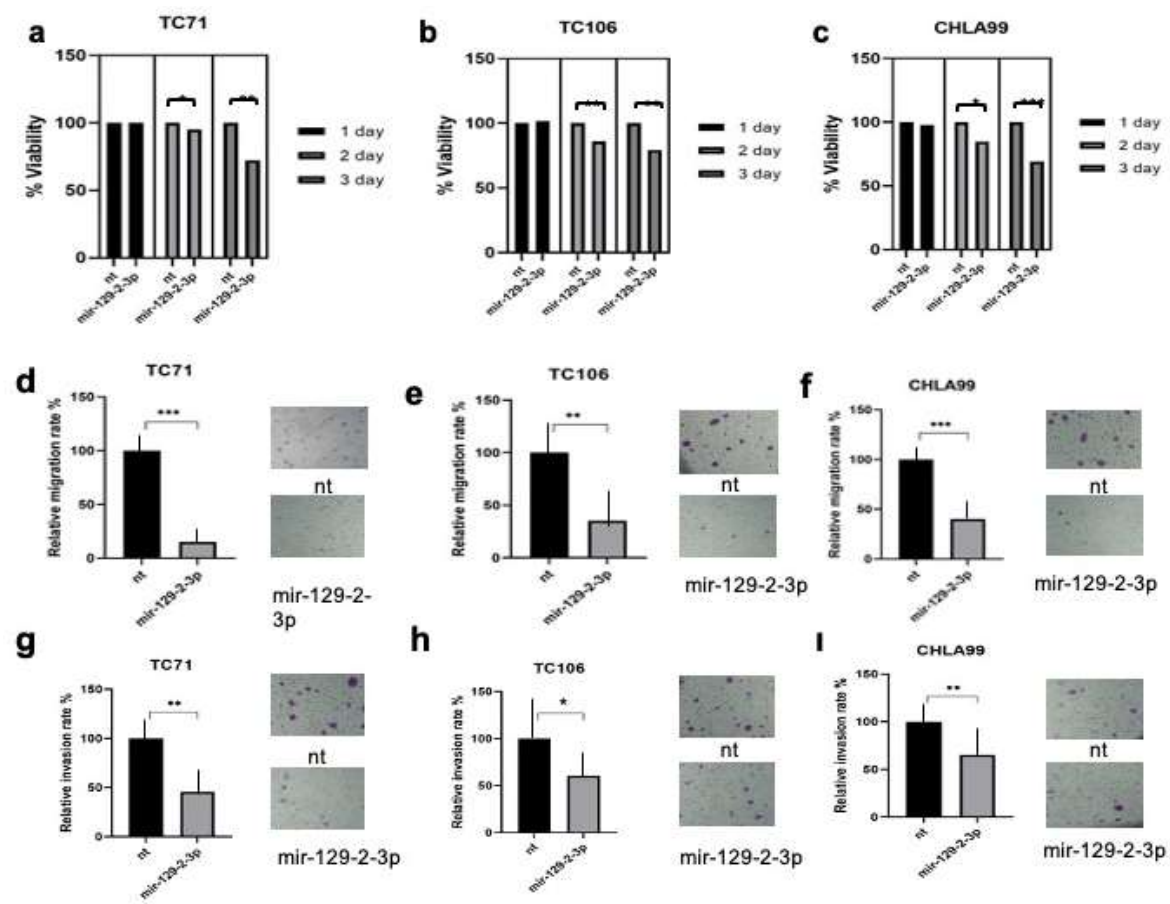

Figure 1. (a-c) Proliferation determined by MTT assay in EWS cell lines with mir-129-2-3p or nontargeting miRNA. A significant decrease is seen in the proliferation rate of the cells transfected with mir-129-2-3p. ; (d-f) Migration and; (gi) invasion assays of EWS cells transfected with miR-129-2-3p mimic and nontargeting miRNA. mir-129-2-3p was reducing migration and invasion capacities in TC-71, TC106, and CHLA99 cells ${ }^{*}, P<0.05 ;{ }^{* *}, P<0.01 ;{ }^{* * *}, P<0.001$. 

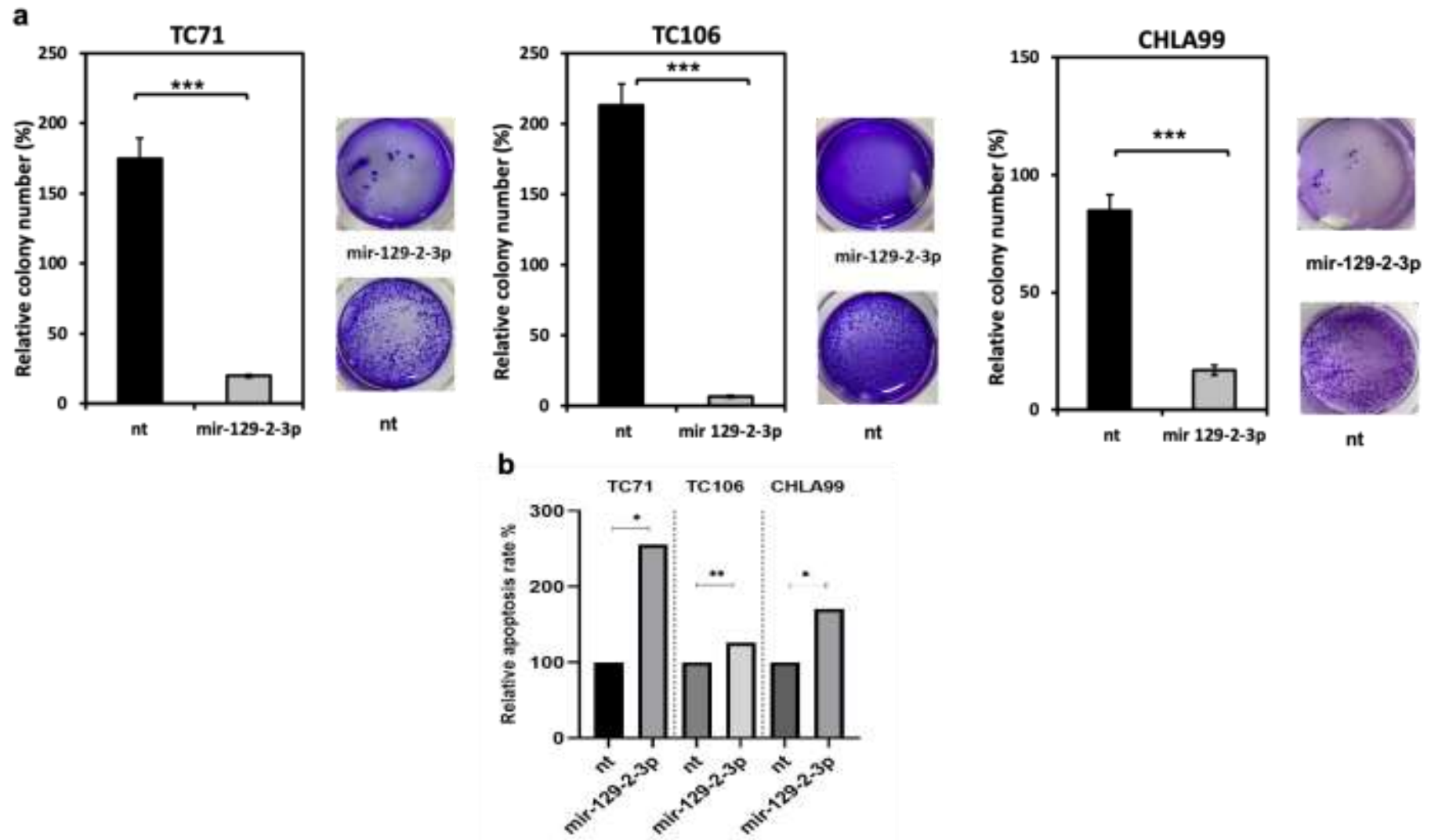

Figure 2. a) Colony formation in TC71, TC106, and CHLA99 treated with mir-129-2-3p or nontargeting. The colony capacity of the 3 cell lines were decreased compared to the control group ${ }^{*}, P<0.05 ;{ }^{* *}, P<0.01 ;{ }^{* * *}, P<0.001$. b) Apoptosis assay of EWS cells transfected with miR-129-2-3p mimic and nontargeting miRNA. miR-129-2-3p transfected cells caused increased in the caspase- 3 activity ${ }^{*}, P<0.05$; ${ }^{*}, P<0.01$.

\section{Identification of target genes of mir-129-2-3p}

It was determined that SOX4 and CDK6, which are the target genes of mir-129-2-3p, were significantly decreased in EWS cells transfected with mir-129-2-3p compared to control cells. The level of SOX4 expression in TC-71, TC-106 and CHLA-99 cells transfected with mir-129-2-3p was calculated as $p<0.05$ in TC-71, $p<0.001$ in TC-106 and $p<0.001$ in CHLA-99 cells. The expression level of CDK6 was found to be $p$ $<0.05$ in TC-71 cell, $p<0.05$ in CHLA-99 and $P<0.001$ in TC-106 cell (Figure 3).
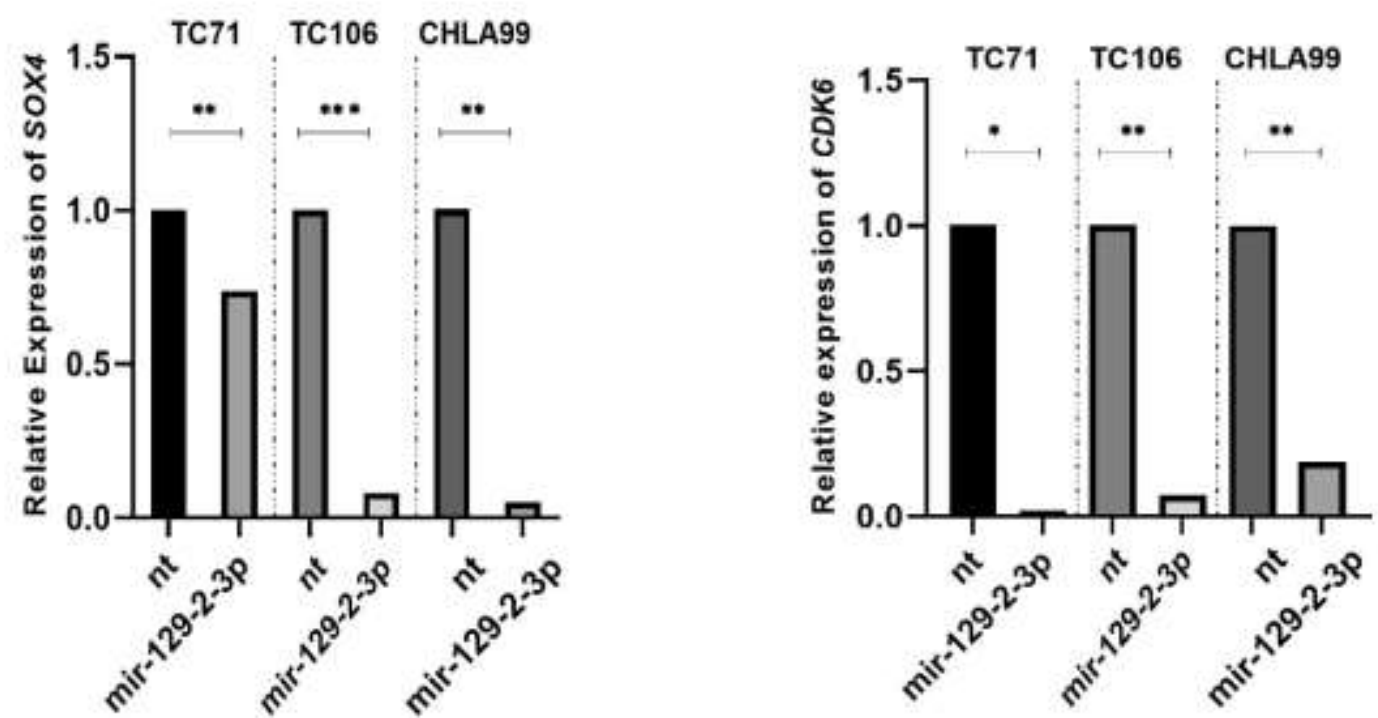

Figure 3. Relative SOX4 and CDK6 expression levels transfected with mir-129-2-3p cells. GAPDH used as internal control. The expression levels of SOX4 and CDK6 were downregulated in mir-129-2-3p cells compared to nt cells. ${ }^{*}, P$ $<0.05 ;{ }^{* *}, P<0.01 ;{ }^{* * *}, P<0.001$. 


\section{Mir-129-2-3p expression levels in EWS tissue samples}

We also performed qRT-PCR to evaluate the expression levels of mir-129-2-3p to determine the differences between EWS tumor tissues and adjacent normal tissues in patients (31 EWS tissues and 12 corresponding adjacent normal tissues). The clinicopathological data of patients were retrieved from medical records, including gender, age, Enneking stage, location and invasive procedures. The association of these variables with mir-129-2-3p are given in Table 1. Of the patients enrolled in the research, 19 were male and 12 were female. Eleven EWS patients were younger than 14 years and the mean age of the patients was 19.5 years. The qRT-PCR data exhibited that the expression level of miR-129-2-3p was significantly downregulated in recurrent EWS samples compared to control samples $(p<0.05)$. To evaluate the correlation of miR-129-2-3p expression in EWS and normal tissue samples, we utilized Receiver Operating Characteristic (ROC) analysis, which demonstrated that miR-129-2-3p expression was statistically significant among the groups (AUC:0.691), (Figure 4).

Table 1. Clinicopathological features of EWS patients and comparison of their mir-129-2-3p expression levels

\section{Characteristics}

\section{Age}

$\leqslant 14$

$>14$

Female

\section{Enneking stage}

IIB

IIIB

\section{Location}

Extremities

Pelvis

Central

\section{Invasive procedure}

Surgery
Resection
Biopsy (JAM+Tru-cut)
Excisional biopsy

$\%$ mir-129-2-3p ( $p$ value)

35,5

64,5

61,3

38,7

77,4

0,03

22,6

61,3

29

9,7
6,5

22,6

64,5

6,5 
a

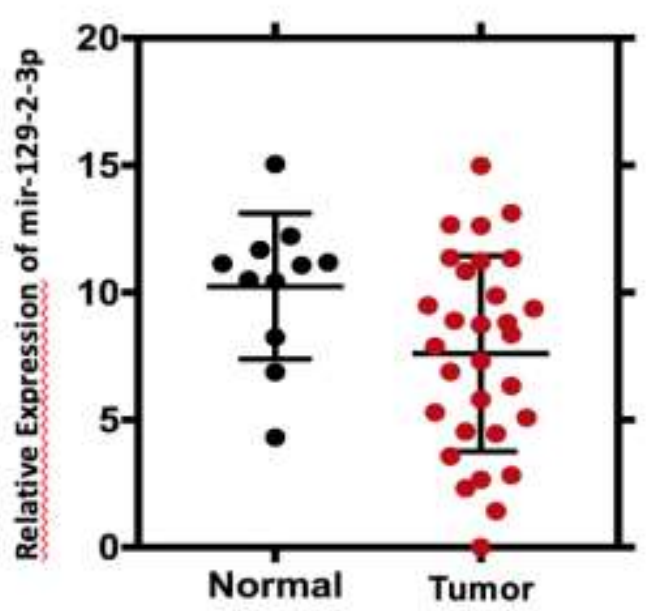

b

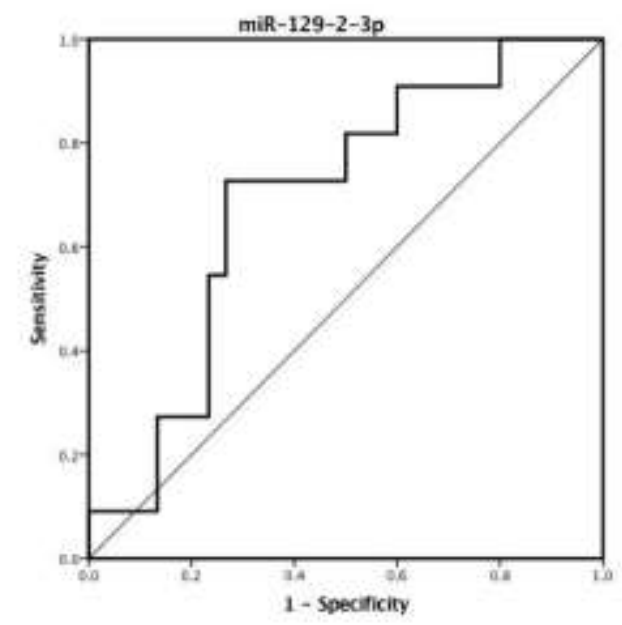

Figure 4. Endogenous relative expression of mir-129-2-3p by qRT-PCR in corresponding tissue and Ewing sarcoma patients. a) Representation of mir-129-2-3p expression in normal and tumor samples $(P<0.05)$ b) Representation of the ROC curve for mir-129-2-3p. RNU43 used as internal control. (AUC=0.691).

\section{DISCUSSION}

Up to date, this is the first paper which evaluates the regulatory role of mir-129-2-3p on EWS cell lines and human EWS tissue samples. In recent years, although more treatment alternatives were developed for EWS, most of the treatment options provide insufficient outcomes since EWS is a heterogeneous cancer type [11] . Despite efforts to improve treatment methods in recent years, the survival rate for this cancer is still low. Ewing Sarcoma tumor has the ability to resist traditional treatments and to renew itself. While responses to chemotherapeutic treatments are sometimes very good, they are inadequate for some patients [12]. After transfection of mir-129-2-3p, which acts as a tumor suppressor, into EWS cells, there was a significant decrease in the proliferation, invasion, migration and cloning properties of the cells, and an increase in apoptosis characterization. Thus, functional studies confirmed that mir-129-2-3p plays an effective role in the progression of EWS.

The expression levels of CDK6 and SOX4 genes, which are target genes of mir-129-2-3p (determined by in silico analysis), showed a significant decrease in the transfected cell lines. The expression of mir-1292-3p was significantly decreased in EWS tumor samples, and this downregulation was more obvious in Enneking IIB patients. The tumor suppressor effect of mir-129-2-3p was shown in ovarian cancer [13], cholangiocarcinoma [14], gastric cancer [15], glial and glioneuronal tumors [16]. It was found that mir-129-3p is suppressed by DNA hypermethylation in primary gastric cancers, and its decreased expression level is associated with the clinical and pathological characteristics of individuals with gastric cancer [17]. It was found that the level of mir-129-2-3p had lower expression in gastric cancer samples and breast cancer cell lines compared to healthy individuals [18-20]. miR-129-2-3p is computationally predicted and functionally validated to function as upstream regulators of SOX4 [18]. We also searched for potential target sequences in the 3'UTR of SOX4 and CDK6 using in silico programs, miRanda, TargetScan, and miRbase. Putative binding sites were found in miR-129-2-3p. There is evidence that cyclin-dependent kinase 6 (CDK6), a cell proliferation regulator, is a direct target of mir-129-2-3p. Mir-129-2-3p, responsible for decreased CDK6 expression, plays a pivotal role in the regulation of cell proliferation [21]. By affecting the reduction in regulation of mir-129-2-3p in cancer cells, it results in the proliferation of cancer cells. On the contrary, the deregulation of mir-129-2-3p results in an increase in the proliferation of endometrial tumor cells as a result of the decrease in the level of CDK6, which is the target gene [22]. In a study of neurotensin and its primary receptor, NTSR1, which cause proliferation of glioblastoma cells in knockout mice, expressions of miR-29b1 and mir-129-3p, which are effective on CDK6 gene expression, increased [21]. It was suggested that mir$129-2-3 p$ could be used as a promising prognostic marker for differentiation of benign tumor and normal tissues in renal cell carcinoma [23]. SOX4, one of the other genes targeted by mir-129-2-3p, is an oncogene with deregulation detected in many SRY-related cancers [24]. SOX4, the gene affected by the abnormal expression of mir-129-2-3p which contributes to the proliferation of tumor cell lines, is among the factors that play a role in the transmission of the Wnt signal that is effective in cancer development. SOX4 is associated with transforming growth factor $\beta$ (TGF $\beta$ ), Hedgehog, and Notch pathways [25]. In endometrial cancer and 
gastric cancers, there is a clear link between epigenetic factors and overexpression of mir-129-2-3p and SOX4. In experiments where mir-129-2-3p levels returned to normal in cancer cells, the expression of SOX4 was decreased. Furthermore, it was proposed that the promoter hypermethylation of mir-129-2-3p is the main factor contributing to the overexpression of SOX4 in some cancer types [17]. It was reported that it is one of the factors that leads to tumorigenesis by increasing the expression of SOX4, the target of mir-129-2-3p, in the development of hepatocellular cancers [26]. Mir-129-2-3p was shown to target genes associated with multiple metastases, including SOX4, in renal cell carcinomas [23]. In our study, it was revealed for the first time in the literature that mir-129-2-3p levels were expressed differently in Ewing Sarcoma cell lines TC-71, TC-106 and CHLA-99, and mir-129-2-3p can be used in the diagnosis of cases with Ewing Sarcoma.

In this study, the role of mir 129-2-3p in the pathogenesis and progression of Ewing Sarcoma was demonstrated functionally. Based on the conclusion that aggressive Ewing Sarcoma cell lines transfected with mir-129-2-3p become less aggressive, the use of mir-129-2-3p in the clinical treatment of Ewing Sarcoma cases may be considered.

Funding: This research was funded by by Istanbul University, Scientific Research Projects (Grant No: 21573)

Conflicts of Interest: The authors declare no conflict of interest.

\section{REFERENCES}

1. Applebaum MA, Goldsby R, Neuhaus J, DuBois SG. Clinical features and outcomes in patients with secondary Ewing sarcoma. Pediatr Blood Cancer. 2013;60(4):611-5.

2. Petersen JA. Early warning score challenges and opportunities in the care of deteriorating patients. Dan Med J. 2018;65(2).

3. Samuel G, Crow J, Klein JB, Merchant ML, Nissen E, Koestler DC, et al. Ewing sarcoma family of tumors-derived small extracellular vesicle proteomics identify potential clinical biomarkers. Oncotarget. 2020;11(31):2995-3012.

4. Guzel E, Okyay TM, Yalcinkaya B, Karacaoglu S, Gocmen M, Akcakuyu MH. Tumor suppressor and oncogenic role of long non-coding RNAs in cancer. North Clin Istanb. 2020;7(1):81-6.

5. Lee YS, Dutta A. MicroRNAs in cancer. Annu Rev Pathol. 2009;4:199-227.

6. Guzel Tanoglu E, Ozturk S. miR-145 suppresses epithelial-mesenchymal transition by targeting stem cells in Ewing sarcoma cells. Bratisl Lek Listy. 2021;122(1):71-7.

7. Tian XY, Zhang L, Sun LG, Li M. Epigenetic Regulation of miR-129-2 Leads to Overexpression of PDGFRa and FoxP1 in Glioma Cells. Asian Pac J Cancer Prev. 2015;16(14):6129-33.

8. Xiao $\mathrm{Y}$, Li X, Wang H, Wen R, He J, Tang J. Epigenetic regulation of miR-129-2 and its effects on the proliferation and invasion in lung cancer cells. J Cell Mol Med. 2015;19(9):2172-80.

9. Yang $Y$, Huang JQ, Zhang X, Shen LF. MiR-129-2 functions as a tumor suppressor in glioma cells by targeting HMGB1 and is down-regulated by DNA methylation. Mol Cell Biochem. 2015;404(1-2):229-39.

10. Kang M, Li Y, Liu W, Wang R, Tang A, Hao H, et al. miR-129-2 suppresses proliferation and migration of esophageal carcinoma cells through downregulation of SOX4 expression. Int J Mol Med. 2013;32(1):51-8.

11. Riggi N, Suvà ML, Stamenkovic I. Ewing's Sarcoma. N Engl J Med. 2021;384(2):154-64.

12. Van Mater D, Wagner L. Management of recurrent Ewing sarcoma: challenges and approaches. Onco Targets Ther. 2019;12:2279-88.

13. Liu F, Zhao H, Gong L, Yao L, Li Y, Zhang W. MicroRNA-129-3p functions as a tumor suppressor in serous ovarian cancer by targeting BZW1. Int J Clin Exp Pathol. 2018;11(12):5901-8.

14. Chen C, Jiang J, Fang M, Zhou L, Chen Y, Zhou J, et al. MicroRNA-129-2-3p directly targets Wip1 to suppress the proliferation and invasion of intrahepatic cholangiocarcinoma. J Cancer. 2020;11(11):3216-24.

15. Yu X, Luo L, Wu Y, Liu Y, Zhao X, Zhang X, et al. Gastric juice miR-129 as a potential biomarker for screening gastric cancer. Med Oncol. 2013;30(1):365.

16. Ames HM, Yuan M, Vizcaíno MA, Yu W, Rodriguez FJ. MicroRNA profiling of low-grade glial and glioneuronal tumors shows an independent role for cluster 14q32.31 member miR-487b. Mod Pathol. 2017;30(2):204-16.

17. Shen R, Pan S, Qi S, Lin X, Cheng S. Epigenetic repression of microRNA-129-2 leads to overexpression of SOX4 in gastric cancer. Biochem Biophys Res Commun. 2010;394(4):1047-52.

18. Yu X, Song H, Xia T, Han S, Xiao B, Luo L, et al. Growth inhibitory effects of three miR-129 family members on gastric cancer. Gene. 2013;532(1):87-93.

19. Tang X, Tang J, Liu X, Zeng L, Cheng C, Luo Y, et al. Downregulation of miR-129-2 by promoter hypermethylation regulates breast cancer cell proliferation and apoptosis. Oncol Rep. 2016;35(5):2963-9.

20. Zhu M, Liang $Q$, Chen $T$, Kong $Q, Y e ~ G, Y u ~ S$, et al. Identification and validation of methylated differentially expressed miRNAs and immune infiltrate profile in EBV-associated gastric cancer. Clin Epigenetics. 2021;13(1):22. 
21. Ouyang Q, Chen G, Zhou J, Li L, Dong Z, Yang R, et al. Neurotensin signaling stimulates glioblastoma cell proliferation by upregulating c-Myc and inhibiting miR-29b-1 and miR-129-3p. Neuro Oncol. 2016;18(2):216-26.

22. Wu J, Qian J, Li C, Kwok L, Cheng F, Liu P, et al. miR-129 regulates cell proliferation by downregulating Cdk6 expression. Cell Cycle. 2010;9(9):1809-18.

23. Chen X, Ruan A, Wang X, Han W, Wang R, Lou N, et al. miR-129-3p, as a diagnostic and prognostic biomarker for renal cell carcinoma, attenuates cell migration and invasion via downregulating multiple metastasis-related genes. J Cancer Res Clin Oncol. 2014;140(8):1295-304.

24. Vervoort SJ, Van Boxtel R, Coffer PJ. The role of SRY-related HMG box transcription factor 4 (SOX4) in tumorigenesis and metastasis: friend or foe? Oncogene. 2013;32(29):3397-409.

25. Scharer CD, McCabe CD, Ali-Seyed M, Berger MF, Bulyk ML, Moreno CS. Genome-wide promoter analysis of the SOX4 transcriptional network in prostate cancer cells. Cancer Res. 2009 Jan 15;69(2):709-17.

26. Chen X, Zhang L, Zhang T, Hao M, Zhang X, Zhang J, et al. Methylation-mediated repression of microRNA 129-2 enhances oncogenic SOX4 expression in HCC. Liver Int. 2013;33(3):476-86.

CC (1) \& ${ }^{\circledR} 2021$ by the authors. Submitted for possible open access publication under the terms and conditions of the Creative Commons Attribution (CC BY NC) license (https://creativecommons.org/licenses/by-nc/4.0/). 